\title{
The colonization with Candida species is more harmful in the second trimester of pregnancy
}

\author{
Iris Holzer $^{1} \cdot$ Alex Farr $^{1} \cdot$ Herbert Kiss $^{1} \cdot$ Michael Hagmann $^{2} \cdot$ Ljubomir Petricevic $^{1}$
}

Received: 11 January 2017 / Accepted: 10 February 2017 / Published online: 3 March 2017

(C) The Author(s) 2017. This article is published with open access at Springerlink.com

\begin{abstract}
Purpose Vaginal colonization with Candida species (spp.) during pregnancy has been associated with impaired pregnancy outcomes. There is a reduction in spontaneous preterm birth among women with recurrent asymptomatic colonization of Candida who were treated with clotrimazole. This study aimed to evaluate the impact of the trimester of vulvovaginal colonization with Candida species.

Methods Data from all women, who were tested positive for the vaginal colonization with Candida spp. during the first or second trimester of pregnancy, and who registered for a planned birth at our tertiary referral center between 2005 and 2014 were retrospectively analyzed. Their preterm birth rate served as the primary outcome variable. Secondary outcome variables were neonatal birthweight and Apgar score.

Results Overall, 1066 women were eligible for the study. In 673 women (63\%), who were diagnosed with Candida spp. during the first trimester of pregnancy, the rate of preterm birth was $10 \%(N=64)$. In 393 women $(37 \%)$, who were diagnosed with candidosis during the second trimester, the preterm birth rate was $18 \%(N=71 ; p=0.0002)$. Neonates of women, who presented with vulvovaginal candidosis during the first trimester, had a mean birthweight
\end{abstract}

Ljubomir Petricevic

ljubomir.petricevic@meduniwien.ac.at

1 Division of Obstetrics and Fetomaternal Medicine, Department of Obstetrics and Gynecology, Medical University of Vienna, Waehringer Guertel 18-20, Vienna 1090, Austria

2 Section for Medical Statistics (IMS), Center of Medical Statistics, Informatics and Intelligent Systems, Medical University of Vienna, Vienna, Austria of $3243 \mathrm{~g}$, compared to $2989 \mathrm{~g}$ in the group with a second trimester colonization $(p<0.0001)$.

Conclusion Women who are colonized with Candida spp. during the second trimester of pregnancy have higher rates of preterm birth and lower neonatal birthweight than those who are colonized during the first trimester of their pregnancy. Screening programs for asymptomatic Candida colonization should take this information into account.

Keywords Vaginal smear $\cdot$ Candidosis $\cdot$ Candida colonization $\cdot$ Preterm birth $\cdot$ Birthweight

\section{Introduction}

Preterm birth (PTB) is defined as birth before 37 completed gestational weeks. Despite all efforts in modern maternity care, PTB remains one of the most fundamental challenges in obstetrics, and the leading cause of neonatal morbidity and mortality [1]. Preterm neonates are at an increased risk for a wide range of adverse neonatal outcomes, in addition to the burden of considerable economic consequences that they cause for affected families and health services [2]. Although PTB is considered a multi-factorial event, vaginal infections remain the major associated factor in up to $40 \%$ of all cases with PTB [3-5].

According to Leli et al. [6], the colonization with Candida spp. occurs more frequently in pregnant compared to non-pregnant women (31.4 vs. 19.9\%). In particular, pregnant women are more often suffering from asymptomatic Candida spp. colonization than their non-pregnant counterparts (46.5 vs. 16.0\%) [6]. Apart from bacterial vaginosis, which has extensively been studied for its effect on pregnancy outcomes, there is evidence that even an aerobic bacterial infection during pregnancy should be treated to 
significantly improve perinatal outcomes [7]. The data on the effect of the antenatal vaginal colonization with Candida are still limited [3,6]. Cotch and colleagues found no association of candidosis with low birthweight and PTB [8]. In contrary, our recently published data demonstrated that the recurrent asymptomatic vaginal colonization with Candida spp. in early pregnancy is indeed associated with PTB and low birthweight [9]. This stands in accordance with the results of a study that reported a reduction in spontaneous PTB among women with asymptomatic colonization of Candida spp. who were treated with clotrimazole [10].

Essentially, these results indicate that screening programs for the asymptomatic vaginal colonization with Candida spp. could be of value. However, more data about the ideal time-point and setting of screening programs are needed. To date, it remains unclear whether the primary colonization with Candida during the first or second trimester of pregnancy is more harmful. Our study is the first to evaluate the impact of the trimester of vulvovaginal candidosis in terms of pregnancy outcomes and PTB in particular.

\section{Patients and methods}

Between 1 January 2005 and 1 January 2014, data from all women with singleton pregnancies, who were diagnosed with the vaginal colonization of Candida spp. at the Medical University of Vienna, Department of Obstetrics and Gynecology, were retrospectively analyzed. We considered vaginal smears of both symptomatic and asymptomatic women, who had a first-time Candida colonization during their first or second trimester of pregnancy. Eligible women had singleton pregnancies and registered for a planned birth at our obstetrical center. All of them were antenatally diagnosed with Candida spp. on the basis of their vaginal smears, which were undertaken either during the first or second trimester of pregnancy. Women with bacterial vaginosis, intermediate vaginal flora or other bacterial infections were excluded from the analyses. The standard treatment for Candida vulvovaginitis was local clotrimazole $0.1 \mathrm{~g}$ for 6 days within 3-5 days after diagnosis.

The cut-off between the first and second trimester of pregnancy was determined at $14+0$ gestational weeks with the second trimester being defined as the gestational age between $14+0$ and $28+6$ weeks. Study group 1 consisted of women, who were primarily diagnosed with Candida colonization or candidosis during the first trimester, whereas Study group 2 consisted of women, who were primarily diagnosed with Candida colonization or candidosis during the second trimester of their pregnancy. Pregnancy outcomes were assessed on the basis of gestational age at birth, recorded as term birth at or later than $37+0$ gestational weeks. PTB was defined as the spontaneous birth at or less than $36+6$ gestational weeks. The rate of PTB served as the primary outcome measure. Secondary outcome measures included neonatal birthweight and the neonatal Apgar score. Stillbirth was defined as the term or preterm birth of a neonate that had died in utero and was born with an Apgar score of 0/0/0.

The demographic and background information was summarized and displayed using descriptive statistics. Discrete data are presented as $N(\%)$. The Chi-squared test was used to compare groups of categorical data. For comparison of continuous data, Welch's $t$ test was used. A two-sided $p$ value $<0.05$ was considered statistically significant. Logistic and linear regression was used to determine the adjusted effect of variables. A multiple regression model was conducted that included the following potentially confounding variables that were unequally distributed between the groups and that were compared and considered to have an impact on the end-point: maternal age, tertiary education, history of PTB and nicotine abuse. All relevant data were collected from obstetric databases, patient charts and microbiologic reports.

\section{Results}

Retrospective data analysis identified a total of 1066 women with singleton pregnancies, who were antenatally, and for the first time, diagnosed with asymptomatic and/ or symptomatic Candida spp. on the basis of their vaginal smears, during the first or second trimester of their pregnancy. Out of these women, 673 (63\%) women were diagnosed with Candida spp. during the first trimester, and 393 (37\%) women were diagnosed during the second trimester of pregnancy. Overall, mean maternal age at the time of birth was $30.2 \pm 6.3$ years. Table 1 illustrates the baseline variables, sociodemographic characteristics and obstetrical history of the included study participants. No serious adverse events were reported for the treatment that was subsequently initiated.

In the overall study cohort, the mean gestational age at birth was $38.5 \pm 3.0$ weeks, corresponding to a mean birthweight of $3144 \pm 694 \mathrm{~g}$. In women of study group 1 , the mean gestational age at birth was $38.9 \pm 2.3$ weeks, and in those of study group 2, it was $37.8 \pm 3.9$ gestational weeks. Infants of women in study group 1 had a mean birthweight of $3234 \pm 600 \mathrm{~g}$, compared to a mean of $2989 \pm 810 \mathrm{~g}$ in those who were assigned to study group $2(p<0.0001)$. In study group 1 , the rate of PTB was $10 \%(N=64)$, compared to $18 \%(N=71)$ in study group $2(p=0.0002)$. This was confirmed in a multiple regression model after adjustment 
Table 1 Baseline variables of the 1066 study participants with Candida colonization or candidosis

\begin{tabular}{|c|c|c|c|}
\hline \multirow[t]{3}{*}{ Variable } & \multicolumn{2}{|c|}{ Baseline variables $(N=1066)$} & \multirow{3}{*}{$\begin{array}{l}\text { All } \\
\text { Mean } \pm \mathrm{SD}, N(\%)\end{array}$} \\
\hline & Study group, trimester 1 & Study group, trimester 2 & \\
\hline & Mean $\pm \mathrm{SD}, N(\%)$ & Mean $\pm \mathrm{SD}, N(\%)$ & \\
\hline Participants & $673(63)$ & $393(37)$ & $1066(100)$ \\
\hline Age at birth (years) & $30.4 \pm 6.0$ & $29.8 \pm 6.6$ & $30.2 \pm 6.3$ \\
\hline Nicotine abuse & $144(21)$ & $106(27)$ & $250(23)$ \\
\hline Academic education & $45(7)$ & $34(9)$ & $23(9.7)$ \\
\hline History of PTB & $9(1)$ & $9(2)$ & $18(2)$ \\
\hline
\end{tabular}

$P T B$ preterm birth, $S D$ standard deviation, $N$ number for nicotine abuse as the only remaining significant confounder.

The mean Apgar scores at 1 min were significantly different for neonates of women of study group 1 vs. those of study group $2(8.7 \pm 1.0$ vs. $8.4 \pm 1.6 ; p=0.0004)$. Table 2 summarizes the pregnancy outcomes of the 1066 study participants with Candida colonization.

\section{Discussion}

The present study was undertaken to evaluate the impact of the trimester of Candida colonization with regard to pregnancy outcomes. As it is known that the recurrent asymptomatic colonization with Candida is involved in the multifactorial event of PTB, we now wanted to evaluate the impact of vaginal colonization or infection with Candida spp. on subsequent pregnancy outcomes. This information could help to determine the ideal time-point for a routine antenatal infection-screening program.

It has already been demonstrated that routine antenatal infection-screening programs have the potential to reduce the rates of PTB and late miscarriage in a general population of pregnant women [1]. For this reason, our department has integrated a program for all pregnant women, to screen and treat for the asymptomatic colonization with pathogens, including bacterial vaginosis and vaginal candidosis [11]. As it is known that recurrent vulvovaginal candidosis can be harmful during early pregnancy, the need for early detection and adequate antifungal treatment is clearly emphasized [10].

A recently published review reported that the use of locally delivered dequaliniumchloride led to a complete cure in $84 \%$ of the pregnant women with Candida [12]. Another treatment of vaginitis in pregnancy is octenidine dihydrochloride/phenoxyethanol that has shown a positive effect in women with bacterial vaginosis and/or candidosis [13]. More easily, screening strategies prior to any infection could help balancing the health care budget, as shown in a cost-effectiveness analysis [14]. The costs per prevented PTB or pregnant woman screened amounted to merely $7 \%$ of the direct costs saved as a result of screening. Indeed, PTB incurs notable social and healthcare costs, particularly in European countries with national health care systems

Table 2 Obstetric outcomes of the 1066 study participants with Candida colonization or candidosis

\begin{tabular}{|c|c|c|c|c|c|}
\hline \multirow[t]{3}{*}{ Variable } & \multirow[t]{3}{*}{ Category/unit } & \multicolumn{2}{|c|}{ Study group $(N=1066)$} & \multirow[t]{2}{*}{ All } & \multirow[t]{2}{*}{ Test } \\
\hline & & Trimester 1 & Trimester 2 & & \\
\hline & & Mean $\pm \mathrm{SD}, N(\%)$ & Mean $\pm \mathrm{SD}, N(\%)$ & Mean $\pm \mathrm{SD}, N(\%)$ & $p$ value \\
\hline \multirow[t]{2}{*}{ Pregnancy outcome } & Live birth & $672(100)$ & 388 (99) & $1060(99)$ & NS \\
\hline & Stillbirth & $1(0)$ & $5(1)$ & $6(1)$ & \\
\hline \multirow[t]{2}{*}{ Prematurity } & Preterm birth & $64(10)$ & $71(18)$ & $135(13)$ & 0.0002 \\
\hline & No preterm birth & $609(90)$ & $322(82)$ & 931 (87) & \\
\hline Apgar at $1 \mathrm{~min}$ & Score & $8.7 \pm 1.0$ & $8.4 \pm 1.6$ & $8.6 \pm 1.3$ & 0.0004 \\
\hline Apgar at 5 min & Score & $9.7 \pm 0.9$ & $9.4 \pm 1.6$ & $9.6 \pm 1.2$ & NS \\
\hline Apgar at $10 \mathrm{~min}$ & Score & $9.8 \pm 1.1$ & $9.6 \pm 1.6$ & $9.7 \pm 1.3$ & NS \\
\hline Gestational age at birth & Weeks & $38.9 \pm 2.3$ & $37.8 \pm 3.9$ & $38.5 \pm 3.0$ & NS \\
\hline Birthweight & Grams & $3234 \pm 600$ & $2989 \pm 810$ & $3144 \pm 694$ & $<0.0001$ \\
\hline
\end{tabular}

$N$ number, $S D$ standard deviation, $N S$ not significant 
and socialized medicine. The antenatal infection-screening program that we used in our study has been proven to reduce PTB rates by 50\%, and therewith save these costs $[14,15]$.

In addition to the socioeconomic advantages, the high prevalence of Candida colonization guided us to integrate an antenatal infection-screening program during routine pregnancy care. Data showed that patients with recurrent candidosis during early pregnancy had higher rates of PTB [9]. In the present study, we only analyzed data of women, who were uniformly colonized or affected with Candida spp. and in this patient group, we found an overall PTB rate of $13 \%$. This rate seems to be higher than the PTB rate in a large retrospective Hungarian study that shows a $4.6 \%$ rate of PTB in women, who were colonized with Candida spp. and treated with clotrimazole [16]. The reason for the higher PTB rate in our study might be that our study was conducted in the tertiary setting, including women with high-risk pregnancies and comorbidities that might interfere with the susceptibility for vaginal candidosis. In contrast, the Hungarian study was a nationwide trial that contained mostly data of women with low-risk pregnancies. A randomized controlled trial from Australia [17] reported a PTB rate of $4 \%$ in pregnant women with asymptomatic vaginal Candida colonization who were treated with clotrimazole. Considering the comparable PTB rates among Austrian and Australian women [18, 19], the 4\% PTB rate among the Australian women with candidosis seems to be actually lower than that of our study. The prospective design of the Australian study could have led to the recruitment and randomization of patients without other risks for PTB, since the authors of this study did not clearly list their exclusion criteria.

In 2008, a prospective study analyzed the prevalence of Candida spp. in pregnant women and reported that the highest colonization rate $(65.7 \%)$ was found in women between 21 and 30 years of age [20]. The mean age in our study population was 30.2 years, which seems to be comparable on the basis of our tertiary setting. The mean birthweight of all our study participants with Candida colonization was $3144 \mathrm{~g}$ at a mean gestational age at birth of 38.5 gestational weeks. This seems to be comparable to the mean birthweight of $3330 \mathrm{~g}$ in the nationwide Hungarian trial [16]. Here, the authors reported a mean gestational age of 39.8 weeks at birth, which might be explained by the higher rate of cesarean sections in our tertiary referral center.

The main finding of our study was that the primary colonization with Candida spp. was more harmful in the second than in the first trimester of pregnancy. This finding was highly statistically significant in a multiple regression model. In a large prospective study from Belgium, it was demonstrated that women with a normal vaginal flora in the first trimester of pregnancy had a $75 \%$ lower risk of preterm birth before $35+0$ weeks of gestation, when being compared to women with an abnormal flora [21]. In the meanwhile, the PTB-risk increasing effect of bacterial vaginosis and recurrent Candida colonization during the first trimester of pregnancy was described [9]. The finding of our study, that the occurrence of Candida spp. during the second trimester of pregnancy also influences pregnancy outcomes, and that it is even more harmful during the second trimester of pregnancy, has not yet been described in the literature. It seems to be of particular interest for the planning and organization of infection screening-programs aiming to detect vulvovaginal candidosis, even after first trimester of pregnancy.

According to a recent study on vulvovaginitis in pregnant women, only $7 \%$ of infections are occurring in the first, but $60 \%$ in the second trimester [22]. However, the data of this study included a variety of pathological conditions, and not only the colonization with Candida. Theoretically, immunologic alternations during pregnancy could have contributed to the altered severity and susceptibility of pathogen colonization during pregnancy. Aspects of innate immunity are enhanced towards late pregnancy, so that this may contribute to an immune down-regulation of the physiologic lower genital tract [23]. This could be one of the reasons for the increased detection of vaginal infections during the second trimester of pregnancy. To prevent PTB, Roberts et al. [17] decided to screen and treat for candidosis in the early to mid second trimester of pregnancy. We suggest that this is the ideal time-point for an antenatal infectionscreening program.

It should clearly be stated that our study has a number of limitations. Other factors that occurred after the screening could have contributed to the reported pregnancy outcomes. Moreover, we did not distinguish between moderate and heavy colonization in our study, as Roberts et al. [17] did it in their large controlled trial. Furthermore, we were not able to perform PCR methods. We have to admit that this was not possible due to the retrospective design of our study, which clearly limits its value.

We are also aware that it would have been ideal to differentiate between different Candida spp., as it was done in some previous studies [16, 17]. However, we know that C. albicans is the most frequent species in $80-90 \%$ of all women who suffer from candidosis [20].

\section{Conclusion}

In this study, we have demonstrated that women who are colonized with Candida spp. during the second trimester of pregnancy have higher rates of preterm birth and lower neonatal birthweight than those who are colonized during 
the first trimester of their pregnancy. Despite all limitations, our findings lend support to the idea that time-point of Candida diagnosis and treatment matters. As recurrent candidosis is known to increase the risk of PTB, our results indicate that the antenatal screening for Candida should ideally be performed during the early second trimester of pregnancy. We suggest there is a need for adequate infection screening programs in routine pregnancy care, which should take this information into account.

\section{Compliance with ethical standards}

Author contributions IH, HK and LP designed the study; IH and $\mathrm{AF}$ collected the data; $\mathrm{MH}$ analyzed the data; $\mathrm{IH}, \mathrm{AF}, \mathrm{HK}$ and $\mathrm{LP}$ wrote the paper.

Informed consent The institutional review board approved this study and required neither patient approval nor informed consent for our retrospective analysis of data that were obtained using a standard of care clinical protocol.

\section{Funding None.}

Conflict of interest The authors declare that they have no conflict of interest.

Open Access This article is distributed under the terms of the Creative Commons Attribution 4.0 International License (http:// creativecommons.org/licenses/by/4.0/), which permits unrestricted use, distribution, and reproduction in any medium, provided you give appropriate credit to the original author(s) and the source, provide a link to the Creative Commons license, and indicate if changes were made.

\section{References}

1. Kiss H, Petricevic L, Husslein P (2004) Prospective randomised controlled trial of an infection screening programme to reduce the rate of preterm deliveries. BMJ 329(7462):371

2. Petrou $S$ (2005) The economic consequnces of preterm birth during the first 10 years of life. BJOG 112(Suppl 1):10-15

3. Leitich H, Bodner-Adler B, Brunbauer M, Kaider A, Egarter C, Husslein P (2003) Bacterial vaginosis as a risk factor for preterm delivery: a meta-analysis. Am J Obstet Gynecol 198(1):139-147

4. Goldenberg RL, Cuhone JF, Ions JD, Romero R (2008) Epidemiology and causes of preterm birth. Lancet 371(9606):75-84

5. Romero R, Espinoza J, Kusanovic JP, Gotsch F, Hassan S, Erez O (2006) The preterm paturition syndrome. BJOG 113(Suppl 3): $17-42$

6. Leli C, Menaccit A, Meucci M (2013) Association of pregnancy and candida vaginal colonization in women with or without symptoms of vulvovaginitis. Miner Ginecol 65(3):303-309

7. Han C, Wu W, Fan A, Wang Y, Zhang H, Chu Z, Wang C, Xue F (2015 Feb) Diagnostic and therapeutic advancements for aerobic vaginitis. Arch Gynecol Obstet 291(2):251-257
8. Cotch MF, Hillier SL, Gibbs RS, Eschenbach DA (1998) Epidemiology and outcomes associated with moderate to heavy candida colonization during pregnancy. Vaginal Infections and Prematurity Study Group. Am J Obstet Gynecol 178(2):374-380

9. Farr A, Kiss H, Holzer I, Husslein P, Hagmann M, Petricevic $L$ (2015) Effect of asymptomatic vaginal colonization with Candida albicans on pregnancy outcome. Acta Obstet Gynecol Scand 94(9):989-996

10. Roberts CL, Algert CS, Richard KL, Morris JM (2015) Treatment of vaginal candidiasis for prevention of preterm birth: a systematic review and meta-analysis. Syst. Rev 4:31

11. Farr A, Kiss H, Hagmann M, Marschalek J, Husslein P, Petricevic $L$ (2015) Routine use of an antenatal infection screen-andtreat program to prevent preterm birth: long-term experience at a Tertiary Referral Center. Birth 42(2):173-180

12. Mendling W, Weissenbacher ER, Gerber S, Prasauskas V, Grob $P$ (2016) Use of locally delivered dequalinium chloride in the treatment of vaginal infections: a review. Arch Gynecol Obstet 293(3):469-484

13. Navkov Mikic A, Stojic S (2015) Study results on the use of different therapies for treatment of vaginitis in hospitalized pregnant women. Arch Gynecol Obstet 292(2):271-276

14. Kiss H, Pichler E, Petricevic L, Husslein P (2006) Cost effectiveness of a screen-and-treat program for asymptomatic vaginal infections in pregnancy: towards a significant reduction in the costs of prematurity. Eur J Obstet Gynecol Reprod Biol 127(2):198-203

15. Kiss H, Petricevic L, Martina S, Husslein P (2010) Reducing the rate of preterm birth through a simple antenatal screen-and-treat programme: a retrospective cohort study. Eur J Obstet Gynecol Reprod Biol 153(1):38-43

16. Czeizel AE, Fladung B, Vargha P (2004) Preterm birth reduction after clotrimazole treatment during pregnancy. Eur J Obstet Gynecol Reprod Biol 116(2):157-63

17. Roberts CL, Rickard K, Kotsiou G, Morris JM (2011) Treatment of asymptomatic vaginal candidiasis in pregnancy to prevent preterm birth: an open-label pilot randomized controlled trial. BMC Pregnancy Childbirth 11:18

18. Delnord M, Blondel B, Zeitlin J (2015) What contributes to disparities in the preterm birth rate in European countries. Curr Opin Obstet Gynecol 27(2):133-142

19. Xu XK, Wang YA, Li Z, Lui K, Sullivan EA (2014) Risk factors associated with preterm birth among singletons following assisted reproductive technology in Australia 2007-2009-a population-based retrospective study. BMC Pregnancy Childbirth 14:406

20. Akinbiyi AA, Watson R, Feyi- Waboso P (2008) Prevalence of Candida albicans and bacterial vaginosis in asymptomatic pregnant women in South Yorkshire, United Kingdom. Outcome of a prospective study. Arch Gynecol Obstet 278(5):463-466

21. Donders GG, Van Calesteren K, Bellen G, Reybrouck R, Van den Bosch T, Riphagen I, Von Lierde S (2009) Predictive value for preterm birth of abnormal vaginal flora, bacterial vaginosis and aerobic vaginitis during the first trimester of pregnancy. BJOG 116(10):1315-1324

22. Mucci MJ, Cuestas ML, Cervetto MM, Landaburu MF, Mujica MT (2016) A prospective observational study of vulvovaginitis in pregnant women in Argentina, with special reference to Candidiasis. Mycoses 59(7):429-435

23. Aguin TJ, Sobel JD (2015) Vulvovaginal candidiasis in pregnancy. Curr Infect Dis Rep 17(6):462 